\title{
Case Report \\ Unusual Course of Scimitar Syndrome Preceded by Lung Hypoplasia
}

\author{
Yusuke Hoshino $(D)$ and Junichi Arai \\ Department of Neonatology, Ibaraki Children's Hospital, 3-3-1 Futabadai, Mito-city, Ibaraki 311-4145, Japan \\ Correspondence should be addressed to Yusuke Hoshino; hoshino-tuk@umin.ac.jp
}

Received 6 September 2019; Revised 28 November 2019; Accepted 11 December 2019; Published 20 December 2019

Academic Editor: Jaroslav Hruda

Copyright (c) 2019 Yusuke Hoshino and Junichi Arai. This is an open access article distributed under the Creative Commons Attribution License, which permits unrestricted use, distribution, and reproduction in any medium, provided the original work is properly cited.

In patients with Scimitar syndrome, right pulmonary artery hypoplasia is considered to lead to right lung hypoplasia because of decrease in blood flow. However, there are no reports wherein the change was actually detected. Thus, the exact developmental mechanism of right pulmonary artery hypoplasia and right lung hypoplasia in patients with Scimitar syndrome is unclear. We experienced a case of Scimitar syndrome preceding right lung hypoplasia, and right pulmonary artery hypoplasia gradually revealed with time. We hypothesized that, in our patient, the lung hypoplasia led to pulmonary artery hypoplasia due to decrease in blood flow. If there are no differences in the diameter of the left and right pulmonary artery in patients with Scimitar syndrome at birth, we propose the necessity of careful observation due to the possibility that pulmonary artery hypoplasia may develop in the future.

\section{Introduction}

Scimitar syndrome is a rare disease characterized by partial anomalous pulmonary venous drainage from the right lung into the inferior vena cava, hypoplasia of the right lung, and dextroposition of the heart. Patients with Scimitar syndrome have varied clinical presentations and are often diagnosed during adulthood because of the absence of symptoms. The exact developmental mechanism of right pulmonary artery hypoplasia and right lung hypoplasia in Scimitar syndrome is unclear [1]. Moreover, previous reports have not fully discussed the association between Scimitar syndrome and hemodynamic changes after birth. Herein, we report a case of Scimitar syndrome wherein differences were noted between the left and right pulmonary artery diameters after birth. Since this patient was admitted due to premature birth and regularly received chest radiography and cardiac ultrasonography during hospitalization, we were able to observe the changes associated with Scimitar syndrome by chance. We hypothesized that the disease follows a novel course based on hydrodynamic characteristics that explain how right pulmonary artery hypoplasia developed due to right lung hypoplasia.
Also, we obtained consent from the patient's parents with oral and written agreement.

\section{Case Presentation}

A male premature newborn infant was born via vaginal delivery at 31 weeks of gestation with a birth weight of $1,532 \mathrm{~g}$. Antenatal ultrasound findings were normal. After birth, he experienced respiratory failure and required respiratory support with intubation. He was diagnosed with neonatal respiratory distress syndrome and treated with surfactant administration. In radiography, the right lung was less radiolucent than the left lung, and mesocardia was noticed; he was accordingly diagnosed with right lung hypoplasia. There were no differences between the left and right pulmonary artery diameters at that time.

He regularly underwent chest radiography and cardiac ultrasonography during hospitalization. Serial examinations showed that the heart position had changed from the median position to the right position (Figure 1); the differences between the left and right pulmonary artery diameters gradually became apparent (Figure 2). At 77 days of life (40 weeks postmenstrual age), the right pulmonary artery 

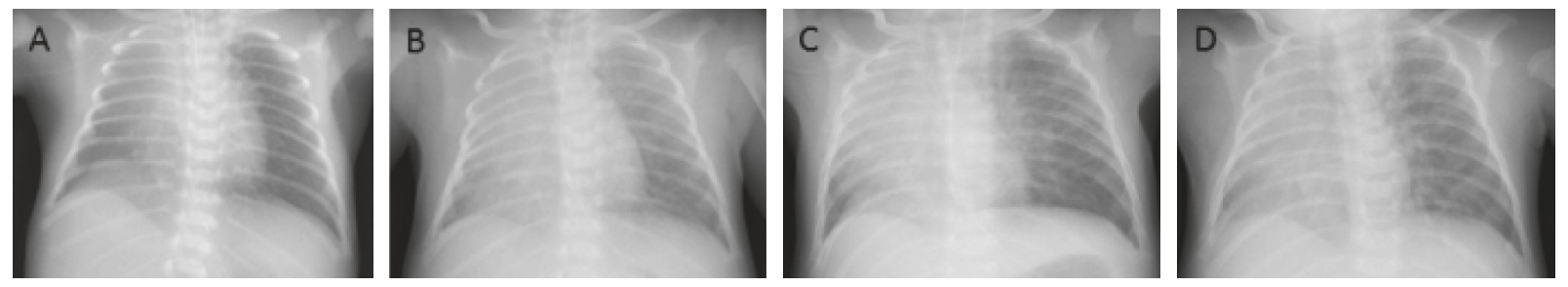

Figure 1: (a) Chest radiography image obtained at 1 day of life showing right lung hypoplasia and mesocardia. (b) Chest radiography image obtained at 14 days of life, showing that the heart had shifted slightly to the right side. (c) Chest radiography image obtained at 45 days of life showing that the heart had further shifted to the right side. (d) Chest radiography image obtained at 77 days of life (40 weeks postmenstrual age), the heart was completely located in the right thoracic cavity.
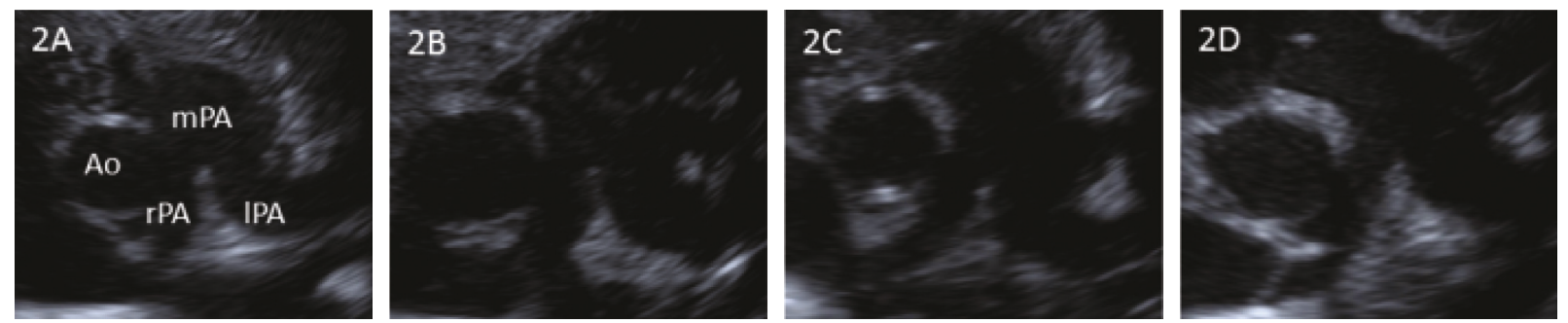

Figure 2: (a) No difference between the left and right pulmonary artery diameter noted at 1 day of life. (b) At 14 days of life, the right pulmonary artery (rPA) diameter was $3.0 \mathrm{~mm}$, and the left pulmonary artery (lPA) diameter was $3.6 \mathrm{~mm}$. (c) At 45 days of life, the rPA diameter was $3.1 \mathrm{~mm}$, and the 1PA diameter was $6.2 \mathrm{~mm}$. (d) At 77 days of life (40 weeks postmenstrual age), the rPA diameter was $3.4 \mathrm{~mm}$, and the IPA diameter was $6.8 \mathrm{~mm}$.

diameter was $3.2 \mathrm{~mm}(-2.5 \mathrm{SD})$, whereas the left pulmonary artery diameter was $6.2 \mathrm{~mm}(+2.7 \mathrm{SD})$. Contrast-enhanced computed tomography examination showed partial anomalous pulmonary venous drainage from the right lung into the inferior vena cava at 73 days of life (Figure 3), and he was diagnosed with Scimitar syndrome due to right lung hypoplasia and dextroposition of the heart.

\section{Discussion}

Here, we report an unknown course of Scimitar syndrome. Previous reports suggested that right pulmonary artery hypoplasia leads to right lung hypoplasia because of decrease in blood flow $[2,3]$. However, there have been no reports wherein the change has been actually detected. We hypothesized that lung hypoplasia led to right pulmonary artery hypoplasia associated with Scimitar syndrome after birth.

In our case, right lung hypoplasia occurred first during the fetal period. However, there were no differences between the left and right pulmonary artery diameters at birth (31 weeks of gestation). Shear stress in the tangential direction due to blood flow and extension stretch in the vertical direction due to intravascular pressure are considered to be necessary for blood vessel maturation $[4,5]$. Since the pulmonary circulation had not been established during the fetal period, we considered that there were no differences in maturation between the left and right pulmonary arteries. After pulmonary circulation was established, we considered right lung hypoplasia led to pulmonary artery hypoplasia because of decrease in blood flow.

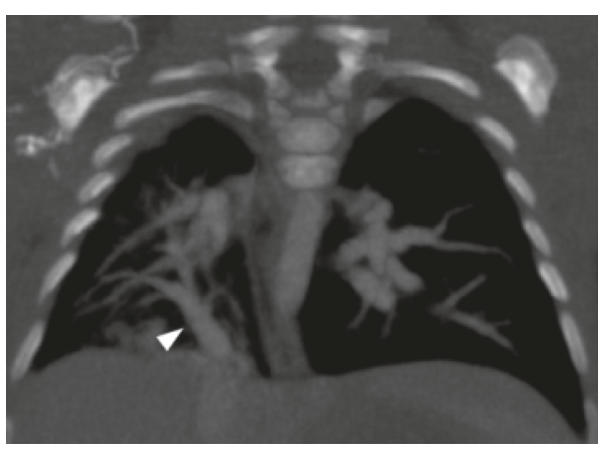

FIgURE 3: Contrast-enhanced computed tomography examination showed partial anomalous pulmonary venous drainage from the right lung into the inferior vena cava (arrow).

There have been several reports in which Scimitar syndrome was diagnosed during the neonatal period $[1,6]$. In these reports, right pulmonary artery hypoplasia did not occur at birth. However, there was a possibility of a future occurrence of pulmonary artery hypoplasia. If there are no differences between the left and right pulmonary artery in patients with Scimitar syndrome at birth, careful observation is necessary because of the possibility that pulmonary artery hypoplasia may develop after birth.

In a case of Scimitar syndrome increasing pulmonary blood flow or showing signs of right heart failure, surgical treatment is considered [7]. In our case, cardiac catheterization showed normal pulmonary-to-systemic blood flow ratio $(\mathrm{Qp} / \mathrm{Qs}$ ratio $=1: 1)$, and we diagnosed for mild pulmonary hypertension. Thus, our patient did not 
undergo surgical treatment and continues with regular follow-up.

\section{Disclosure}

The abstract had been submitted to the 121st Annual Meeting of the Japan Pediatric Society held in Fukuoka on April 20-22, 2018.

\section{Conflicts of Interest}

The authors declare that there are no conflicts of interest regarding the publication of this article.

\section{Acknowledgments}

The authors thank the patient and his family for participating in this work.

\section{References}

[1] A. Lastinger, M. El Yaman, R. Gustafson, and P. Yossuck, "Scimitar syndrome and $H$-type tracheo-esophageal fistula in a newborn Infant," Pediatrics \& Neonatology, vol. 57, no. 3, pp. 236-239, 2016.

[2] R. Van Praagh, S. Van Praagh, P. Vlad, and J. D. Keith, "Anatomic types of congenital dextrocardia," The American Journal of Cardiology, vol. 13, no. 4, pp. 510-531, 1964.

[3] R. P. Grant, "The syndrome of dextroversion of the heart," Circulation, vol. 18, no. 1, pp. 25-36, 1958.

[4] S. Usami, H.-H. Chen, Y. Zhao, S. Chien, and R. Skalak, "Design and construction of a linear shear stress flow chamber," Annals of Biomedical Engineering, vol. 21, no. 1, pp. 77-83, 1993.

[5] N. Shimizu, K. Yamamoto, S. Obi et al., "Cyclic strain induces mouse embryonic stem cell differentiation into vascular smooth muscle cells by activating PDGF receptor $\beta$," Journal of Applied Physiology, vol. 104, no. 3, pp. 766-772, 2008.

[6] G. Shinohara, K. Morita, Y. Uno, M. Yamashiro, and K. Hashimoto, "Scimitar syndrome in an infant with right lung hypoplasia, ventricular septal defect, and severe pulmonary hypertension," General Thoracic and Cardiovascular Surgery, vol. 58, no. 10, pp. 524-527, 2010.

[7] Z. Dokumcu, E. Divarci, and A. Erdener, "Thoracoscopic treatment of left-to-right shunt in a child with scimitar syndrome," Cardiology in the Young, vol. 28, no. 1, pp. 159-162, 2018. 


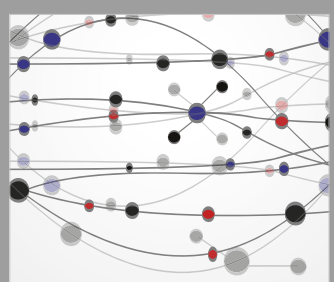

The Scientific World Journal
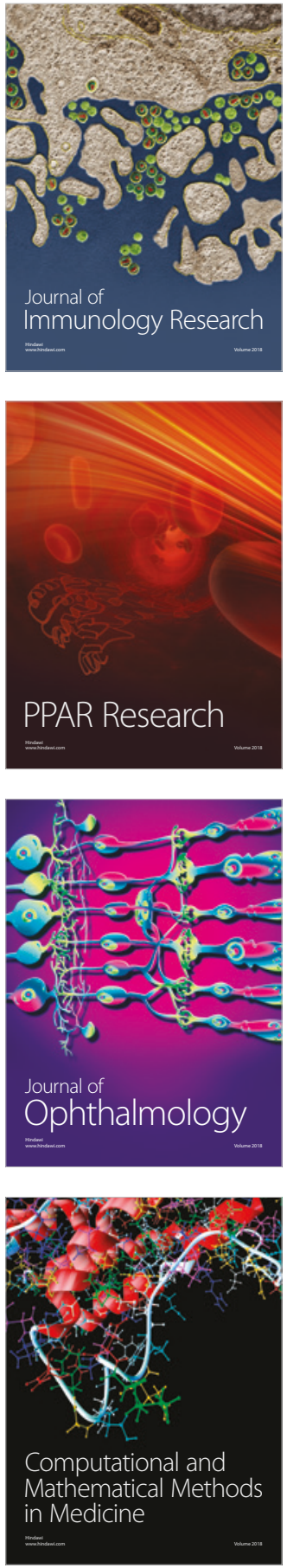

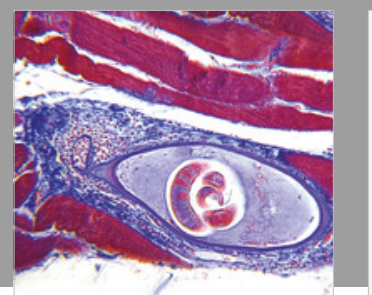

Gastroenterology Research and Practice

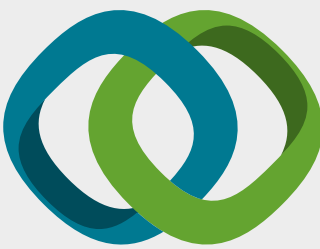

\section{Hindawi}

Submit your manuscripts at

www.hindawi.com
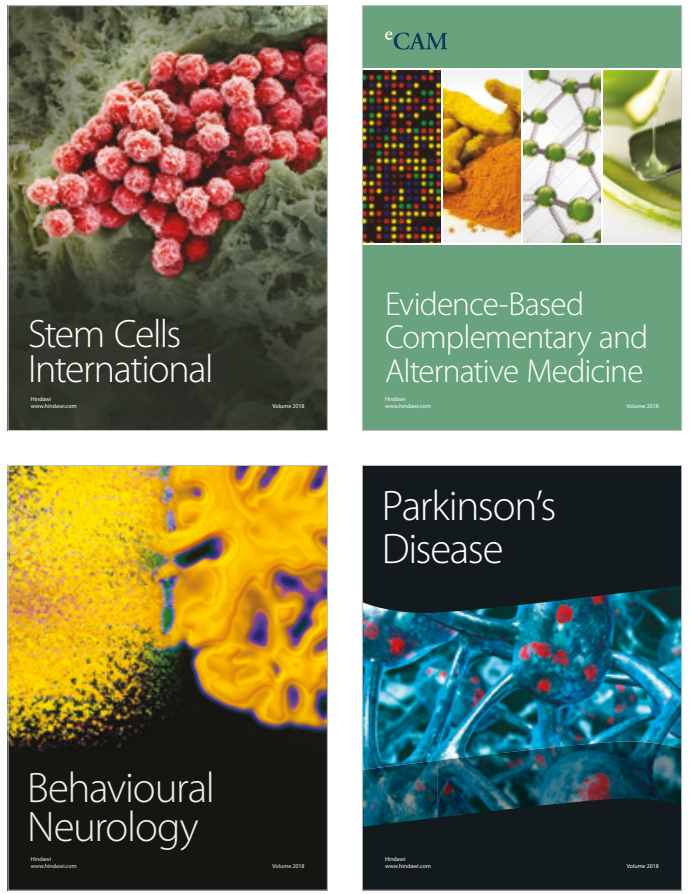

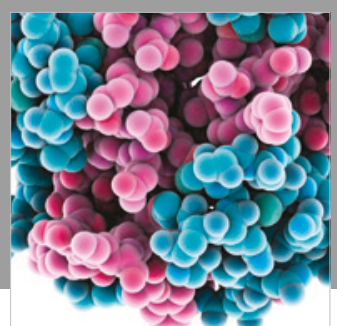

ournal of

Diabetes Research

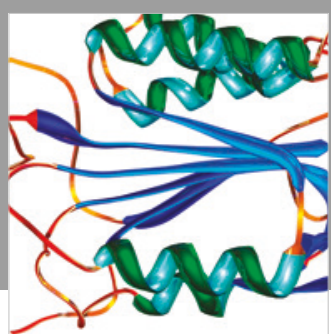

Disease Markers
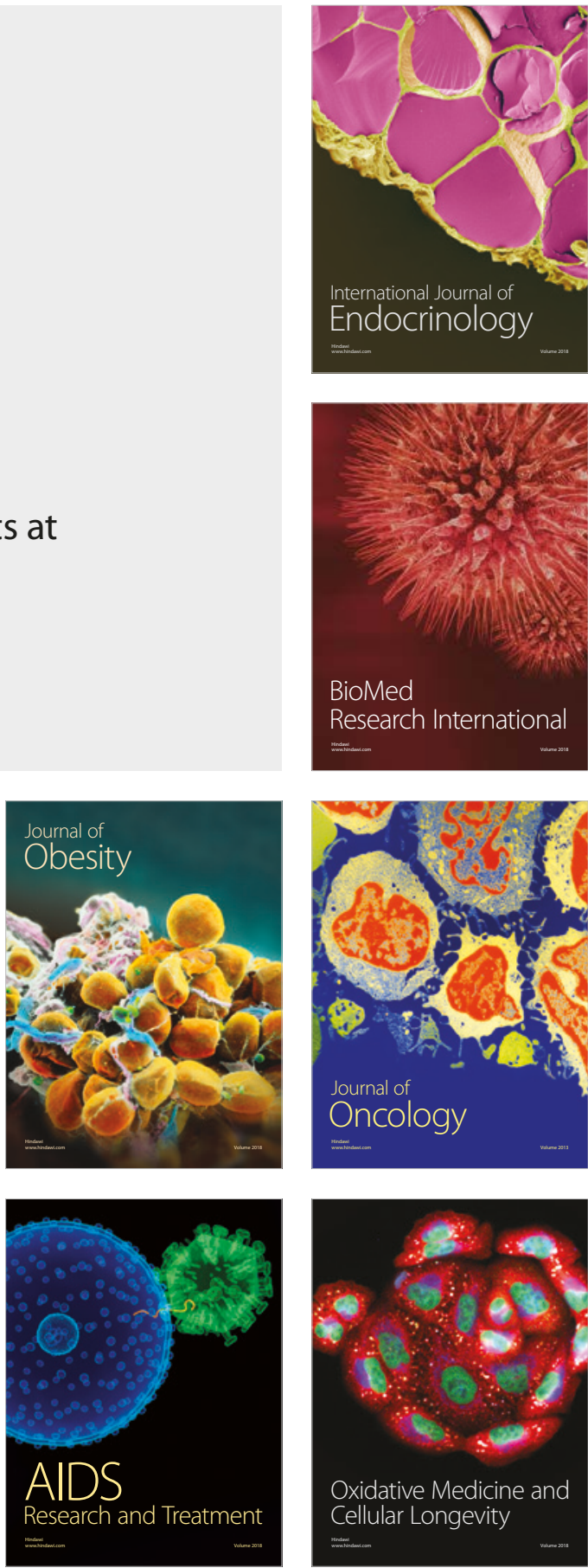